\title{
Synthesis of $\beta$-amino alcohol derivatives from phenols in presence of phase transfer catalyst and lipase biocatalyst
}

\author{
Vasant S. Borude, Rikhil V. Shah and Sanjeev R. Shukla*
}

Dept. of Fibres and Textile Processing Technology, Institute of Chemical Technology (University under Section-3 of UGC Act 1956), Matunga, Mumbai 400019, India

\section{H R O N I C L E}

Article history:

Received June 25, 2012

Received in Revised form

November 6, 2012

Accepted 6 November 2012

Available online

6 November 2012

\section{Keywords:}

Lipase, $\beta$-Amino Alcohol

Phase Transfer Catalyst

Epichlorohydrin

Biocatalyst

1-Butyl-3-methylimidazolium

Chloride

\begin{abstract}
A B S T R A C T
A simple and environmentally friendly reaction has been developed for the first time for onepot synthesis of $\beta$-amino alcohol derivatives from aromatic phenols, epichlorohydrin and amines by using phase transfer catalysts (PTC) and Aspergillus Oryzae lipase biocatalyst. This method provides access to pharmaceutically relevant products in excellent yields with high regioselectivity. The remarkable catalytic activity and reusability of lipase was possible up to four consecutive cycles.
\end{abstract}

\section{Introduction}

The $\beta$-Amino alcohols are present in many biologically active natural products and chiral auxiliaries containing common intermediates ${ }^{1-3}$. They play an increasingly important role in medicinal chemistry, pharmaceuticals and in organic synthesis ${ }^{4-5} \cdot \beta$-Adrenergic blocking agents $(\beta$ blockers) are used in treatment of a wide variety of human disorders like hypertension, sympathetic nervous system, heart failure, cardiac arrhythmias ${ }^{6-7}$ and also as insecticidal agents ${ }^{8}$.

The skeleton of $\beta$-amino alcohols of the type 1 (Fig. 1) is particularly interesting in biologically active pharmaceutical compounds, which are easily available via one-pot multicomponent reaction process. Compounds such as propranolol 2 are used as selective dopamine $\mathrm{D}_{4}$ receptor antagonists 9 . * Corresponding author. Tel: +91 22-2414 5614 E-mail addresses: srshukla19@gmail.com (S. R Shukla) 
Some $\beta$-amino alcohol derivatives 3 prove to be useful as antagonists of the calcium receptor I that inhibits parathyroid hormone secretagogues ${ }^{10}$, other compounds such as practolol $\mathbf{4}$ and celiprolol hydrochloride $\mathbf{5}$ are the drugs belonging to the class of arloxypropanolamine $\mathbf{1}$ useful as $\beta$ blocker $^{11,12}$.

One of the most common methods used for the synthesis of $\beta$-amino alcohols is the direct aminolysis of epoxides using different promoters or catalysts in the presence of conventional solvents. These include calcium trifluoromethanesulfonate ${ }^{13}$, ionic liquids ${ }^{14}$, bismuth triflate $^{15}$, polymer-supported chiral $\mathrm{Co}\left(\right.$ Salen) complexes $^{16}$, copper(II) acetylacetonate $\left(\mathrm{Cu}(\mathrm{acac})_{2}\right)^{17}$, microwave irradiation ${ }^{18}$, etc.

Synthesis of epoxide from phenol and epichlorohydrin by using different methods, catalysts and organic solvents includes microwave irradiation ${ }^{19}$, cesium fluoride ${ }^{20}$, sodium hydride ${ }^{21}$, cesium carbonate $^{22}$ and $\beta$-cyclodextrin ${ }^{23}$ etc.

The emerging area of green chemistry makes use of safer and non toxic materials, leaves no waste to treat, increases energy efficiency, uses renewable feed stock, maximizes atom economy and minimizes the potential for accidents. Biocatalyst provides excellent alternative in organic synthesis. The lipases from Aspergilllus Oryzae as biocatalyst are well established in organic synthesis because of their stability, selectivity and easy availability ${ }^{24-25}$. The use of lipase is well known in Knoevenagel condensation $^{26}$, Mannich reaction ${ }^{27}$, ester hydrolysis ${ }^{28}$, etc. However, its application in the one-pot synthesis of $\beta$-amino alcohol derivatives from aromatic phenols has not been well explored.

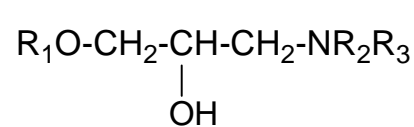

1

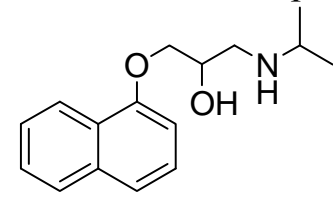

2

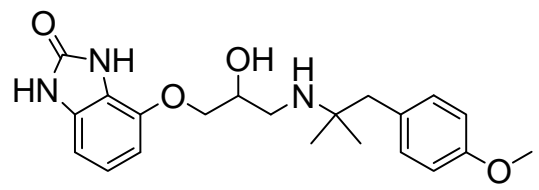

3<smiles>CC(=O)Nc1ccc(OCC(O)CNC(C)C)cc1</smiles>

4<smiles>CCCCN(CC)C(=O)Nc1ccc(OCC(O)CCNCC)c(C(C)=O)c1</smiles>

5

Fig. 1. General formula (1) and examples of biologically active (2-5) $\beta$-amino alcohols

Pchelka et al. ${ }^{19}$ reported the reaction of phenol and epichlorohydrin under microwave irradiation by using PTC like tetrabutylammonium bromide (TBAB). The role of PTC is to facilitate the reaction by migrating a reactant from one phase to another ${ }^{29}$. Commonly used PTCs are the salts of quaternary ammonium or phosphonium compounds, benzyl trimethyl ammonium chloride and $\mathrm{TBAB}^{30}$. Recently ionic liquids (ILs) which are known as environmentally benign and reusable reagents have attracted growing attention due to their high thermal stability ${ }^{31-33}$ ILs based on 1,3dialkylimidazolium cation and pyridine cation are composed of cation/anion combinations, which are similar to the conventional quaternary ammonium salts and hence such type of ILs have the potential for use as $\mathrm{PTC}^{34-36}$.

We herein report the synthesis of pharmaceutically relevant $\beta$-amino alcohol derivatives in onepot reaction along with aromatic phenols, epichlorohydrin and amines by using lipase biocatalyst from Aspergillus Oryzae along with PTC. 


\section{Results and Discussion}

The synthesis of $\beta$-amino alcohol derivatives using Aspergillus Oryzae lipase biocatalyst and PTC has been shown in Scheme 1.

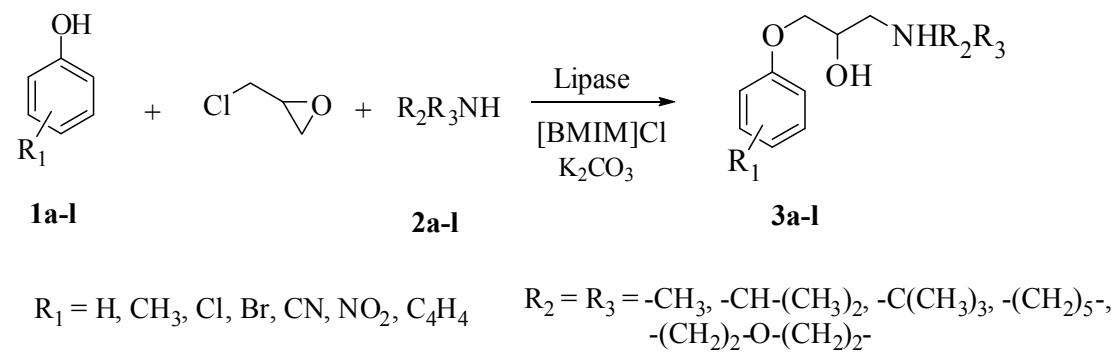

Scheme 1: General Reaction Scheme

The reaction between phenol (1a), epichlorohydrin and amine $\mathbf{2 a}$ was selected as a model reaction for optimizing the reaction parameters such as molar ratio, effects of solvents, catalyst study, catalyst amount, and reusability. As shown in Table 1, entries 1, 2, 3 we carried out the model reaction using different PTC such as triethylamine hydrochloride (TEA.HCl), TBAB and choline chloride. It was observed that the yield of product decreases respectively. The reaction using [BMIM]Cl as PTC gave maximum product yield with less reaction time (Table 1 , entry 5,6 ). This was attributed to small inorganic anion and bulky organic cation of [BMIM]Cl having high stability as compared to choline chloride, TEA.HCl and TBAB. Because the positive charge in [BMIM]Cl is delocalized over two nitrogen atoms and three carbon atoms, it imparts maximum resonance stability as compared to other tetraalkylammonium salts ${ }^{37,38}$.

For further optimization of [BMIM]Cl, the reaction was carried out with different quantities and 0.1 equivalent of [BMIM]Cl with respect to $\mathbf{1 a}$ was found to be optimal (Table 1, entries $4,5,6$ ). In the absence of PTC, the product 3a was afforded in $30 \%$ yield after reaction at $55^{\circ} \mathrm{C}$ for $8 \mathrm{~h}$ (Table 1 , entry 7).

Table 1. Effect of PTC on product yield 3a

\begin{tabular}{cccc}
\hline Entry $^{\text {a }}$ & PTC (equiv.) & Time $(\mathrm{h})$ & ${\text { Yield }(\%)^{\mathrm{b}}}$ \\
\hline 1 & TEA.HCl (0.1) & 5.5 & 55 \\
2 & TBAB (0.1) & 6 & 46 \\
3 & Choline Chloride $(0.1)$ & 6.5 & 42 \\
4 & {$[$ BMIM] Cl (0.05) } & 6 & 41 \\
5 & {$[$ BMIM] Cl (0.1) } & 4 & 60 \\
6 & {$[$ BMIM] Cl $(0.2)$} & 4 & 60 \\
7 & - & 8 & 30 \\
\hline
\end{tabular}

Reaction Condition: ${ }^{a}$ All reactions were carried out with phenol 1a (2 mmol), epichlorohydrin (3 mmol), morpholine 2a $(2 \mathrm{mmol})$, lipase $(10 \% \mathrm{w} / \mathrm{w}, 0.019 \mathrm{~g})$, temperature $\left(55^{\circ} \mathrm{C}\right),{ }^{\mathrm{b}}$ Isolated yields.

For comparison, synthesis of $\beta$-amino alcohol derivatives was also studied using conventional bases such as sodium hydroxide ${ }^{19}(\mathrm{NaOH})$, sodium hydride ${ }^{21}(\mathrm{NaH})$, potassium carbonate $\left(\mathrm{K}_{2} \mathrm{CO}_{3}\right)$ and sodium bicarbonate $\left(\mathrm{NaHCO}_{3}\right)$ (Table 2, entries 1-4). These conventional bases required more reaction time while giving lower product yields. The reaction using Aspergillus Oryzae lipase biocatalyst $(10 \% \mathrm{w} / \mathrm{w})$ gave the product 3a in $60 \%$ yield in $4 \mathrm{~h}$.

Further, excellent results were obtained by using $50 \% \mathrm{w} / \mathrm{w}$ lipase to afford the product $3 \mathrm{a}$ in $86 \%$ yield in $3.5 \mathrm{~h}$ (Table 2, entries 5, 6). Use of $\mathrm{NaHCO}_{3}$ or $\mathrm{K}_{2} \mathrm{CO}_{3}$ as a base in the presence of lipase $(10 \% \mathrm{w} / \mathrm{w})$ as a biocatalyst obtained the product 3a in $87-88 \%$ yield after reacting at $55^{\circ} \mathrm{C}$ for $3.5 \mathrm{~h}$ (Table 2, entries 7,8), since the reaction is promoted by lipase and the base serves to trap the 
resulting hydrochloric acid which is a byproduct of the reaction. In the absence of base, lipase should work as a promoter as well as a captor of the acid. $\mathrm{K}_{2} \mathrm{CO}_{3}$ and $\mathrm{NaHCO}_{3}$ exhibited similar effect on reaction, although among the two, $\mathrm{K}_{2} \mathrm{CO}_{3}$ gave higher product yield as it is more thermally stable and the conjugate base of $\mathrm{K}_{2} \mathrm{CO}_{3}$ is more basic than that of $\mathrm{NaHCO}_{3}{ }^{19,20}$. No reaction could occur at room temperature $\left(35^{\circ} \mathrm{C}\right)$ in the presence of lipase. Reaction also did not occur in the absence of any catalyst.

Lipase biocatalysts are made up of different subunits having high efficiency and selectivity. Therefore, lipase biocatalysts can be used in a variety of organic transformations without the need of additional coenzymes ${ }^{26}$. Different organic solvents were also screened to see their efficiency in the reaction. Lipase is a heterogeneous catalyst and could easily separate from reaction mixture by filtration.

With these results in hand, we developed the one-pot synthesis of $\beta$-amino alcohol derivatives from various substituted aromatic phenols (1a-l), epichlorohydrin and amines (2a-l) to obtain the corresponding products (3a-l) (Table 3$)$. The rate of reaction and product yield was better in case of secondary amines such as morpholine, piperidine compared to primary aliphatic amines like methyl amine, isopropyl amine, isobutyl amine, etc ${ }^{39}$.

The basicity of amine is expected to increase with the number of alkyl groups on the amine. In secondary amine, two alkyl groups are attached directly to the nitrogen atom resulting in better reactivity of the secondary amine as compared to the primary amine. Basicity also depends on stabilization of the conjugate acid formed and the conjugate acid of secondary amine was more stable than primary amine ${ }^{39}$.

Table 2. Effect of catalyst, Base and solvent on product yield 3a

\begin{tabular}{ccccccc}
\hline Entry $^{\mathbf{a}}$ & Catalyst & Base $(1$ equiv. $)$ & Solvent & $\begin{array}{c}\text { Temp. } \\
(\mathrm{C})\end{array}$ & Time (h) & $\begin{array}{c}\text { Yield } \\
(\%)^{\mathbf{b}}\end{array}$ \\
\hline 1 & - & $\mathrm{NaOH}$ & - & 55 & 20 & 40 \\
2 & - & $\mathrm{NaH}$ & - & 55 & 12 & 55 \\
3 & - & $\mathrm{K}_{2} \mathrm{CO}_{3}$ & - & 55 & 15 & 30 \\
4 & - & $\mathrm{NaHCO}_{3}$ & - & 55 & 18 & 25 \\
5 & Lipase $(10 \% \mathrm{w} / \mathrm{w})$ & - & - & 55 & 4 & 60 \\
6 & Lipase $(50 \% \mathrm{w} / \mathrm{w})$ & - & - & 55 & 3.5 & 86 \\
7 & Lipase $(10 \% \mathrm{w} / \mathrm{w})$ & $\mathrm{NaHCO}_{3}$ & - & 55 & 3.5 & 87 \\
8 & Lipase $(10 \% \mathrm{w} / \mathrm{w})$ & $\mathrm{K}_{2} \mathrm{CO}_{3}$ & - & 55 & 3.5 & 88 \\
9 & Lipase $(10 \% \mathrm{w} / \mathrm{w})$ & $\mathrm{K}_{2} \mathrm{CO}_{3}$ & - & 110 & 3 & 35 \\
10 & Lipase $(10 \% \mathrm{w} / \mathrm{w})$ & $\mathrm{K}_{2} \mathrm{CO}_{3}$ & DMF & 55 & 6 & 60 \\
11 & Lipase $(10 \% \mathrm{w} / \mathrm{w})$ & $\mathrm{K}_{2} \mathrm{CO}_{3}$ & Dioxane & 55 & 8 & 50 \\
12 & Lipase $(10 \% \mathrm{w} / \mathrm{w})$ & $\mathrm{K}_{2} \mathrm{CO}_{3}$ & THF & 55 & 7 & 55 \\
\hline
\end{tabular}

Reaction Condition: ${ }^{a}$ All reactions were carried out with phenol 1a $(2 \mathrm{mmol})$, epichlorohydrin $(3 \mathrm{mmol})$, amine 2a (2 mmole), [BMIM]Cl (0.035g, 0.1 equiv.), ${ }^{\mathbf{b}}$ Isolated yields.

In 4-nitrophenol and 4-cyanophenol, due to the presence of electron withdrawing group at para position $^{20,40}$ after formation of phenoxide ion, the loan pair of electron gets stabilized by resonance and hence less available for nucleophilic attack with epichlorohydrin. Therefore, the reaction required more time (Table 3, entries i- 1). 
Table 3. Synthesis of $\beta$-amino alcohol derivatives

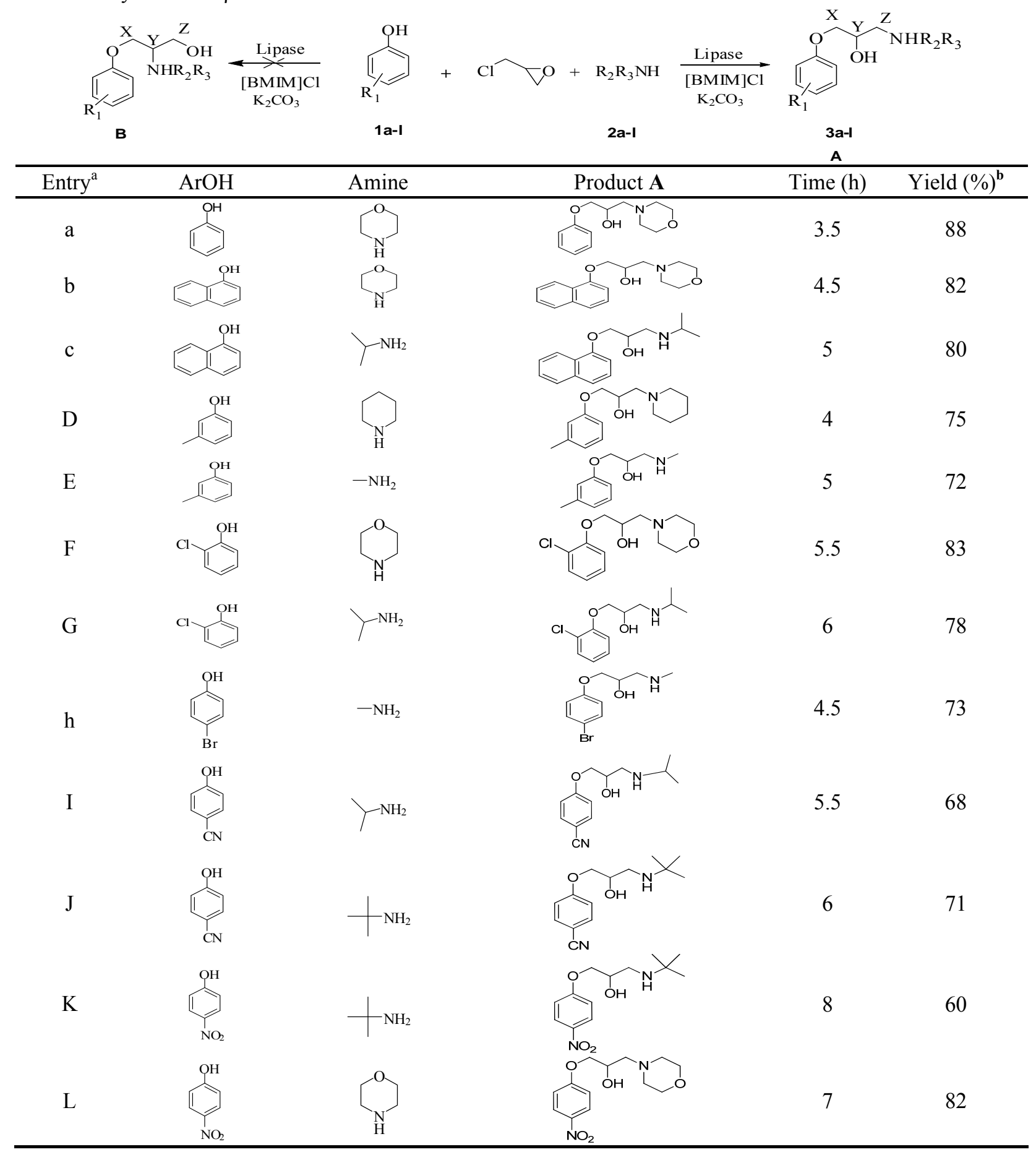

Reaction Condition: ${ }^{\mathrm{a}} \mathrm{All}$ reactions were carried out with phenol 1a-l ( $\left.2 \mathrm{mmol}\right)$, epichlorohydrin ( $\left.3 \mathrm{mmol}\right)$, amine 2a-l (2 $\mathrm{mmol})$, lipase ( $10 \% \mathrm{w} / \mathrm{w}),[\mathrm{BMIM}] \mathrm{Cl}\left(0.1\right.$ equiv.), $\mathrm{K}_{2} \mathrm{CO}_{3}$ (1 equiv.), ${ }^{b}$ Isolated yields. Regioselectivity was determined by NMR spectra.

In 2-chlorophenol, due to the presence of chloro group at ortho position the steric hindrance affects the reaction between phenol and epichlorohydrin, thereby requiring longer reaction time (Table 3, entries $\mathrm{f}, \mathrm{g}$ ). Phenol and its derivatives with electron donating substituents react faster as compared to phenol with electron accepting substituents. Also, the sterically hindered phenol reacts very slowly. 
There are two possible ways of nucleophilic attack with different amines at the epoxide carbon, one at terminal carbon atom to form regioisomer $\mathbf{A}$ and another at internal carbon atom to form regioisomer $\mathbf{B}$ (Table 3). We afforded $\mathbf{A}$ as the major regioisomer, because nucleophilc attack of amines takes place preferentially at the terminal carbon atom of epoxide than internal carbon atom. Regioselectivity was determined by NMR spectrum. Regioisomer $\mathbf{A}$ has secondary alcohol group and carbon attached to that hydroxyl group gives chemical shift at $\delta=68 \mathrm{ppm}$ which we obtained in ${ }^{13} \mathrm{C}$ NMR spectrum of products, while, regioisomer $\mathbf{B}$ has primary alcohol group and carbon atom adjacent to it shows shift at $\delta=60 \mathrm{ppm}$, which was not observed. Thus, the conclusion was that we obtained regioisomer $\mathrm{A}^{39}$. During the recyclability study of the lipase biocatalyst, it was easily separated from the reaction mass by filtration and was recycled up to four times. No significant decrease in the product yield was observed during the first recycle whereas the yield declined up to $70 \%$ after completion of fourth recycle as shown in the Table 4.

Based on the observations, the mechanism for the reaction may be postulated as shown in Scheme 2. The active sites of lipase such as aspartate histidine dyad and oxyanion hole, abstract the acidic proton of the phenols $\mathbf{1 a}-\mathbf{l}$ to form the nucleophiles $\mathbf{X}^{41,42}$, which replace the $\mathrm{Cl}^{-}$of [BMIM]Cl giving the intermediate $\mathbf{Y}$. It reacts with epichlorohydrin to give the intermediate $\mathbf{Z}$. Sequential attack of the amines $\mathbf{2 a -} \mathbf{I}$ on the intermediate $\mathbf{Z}$ gives the final products $\mathbf{3 a -} \mathbf{-}$.

Table 4. Recyclability study of Lipase on product yield 3a

\begin{tabular}{ccc}
\hline Entry $^{\text {a }}$ & Recycle & Yield $^{\mathrm{b}}(\%)$ \\
\hline 1 & - & 88 \\
2 & First & 85 \\
3 & Second & 80 \\
4 & Third & 78 \\
5 & Fourth & 70 \\
\hline
\end{tabular}

Reaction Condition: ${ }^{\mathrm{a}}$ All reactions were carried out with phenol 1a $(2 \mathrm{mmol})$, epichlorohydrin $(3 \mathrm{mmol})$, amine 2a (2 mmole), [BMIM] Cl (0.035g, 0.1 equiv.), $\mathrm{K}_{2} \mathrm{CO}_{3}$ (1 equiv.), ${ }^{\mathrm{b}}$ Isolated yields.
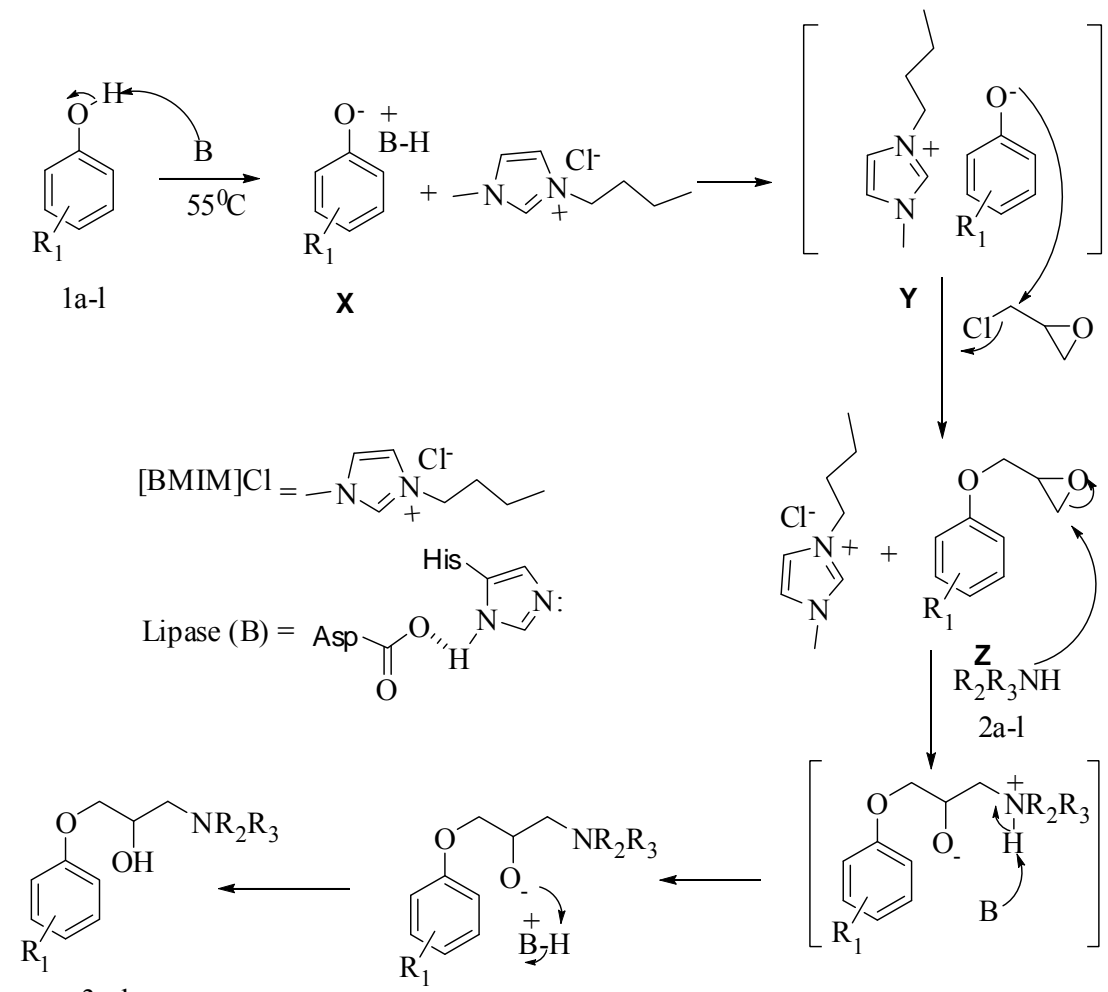
Scheme 2. A plausible reaction mechanism for the formation of $\beta$-amino alcohol derivatives 3a-I.

The FT-IR spectra of synthesized compounds showed the stretching frequency at 1250 and 1040 $\mathrm{cm}^{-1}$ clearly indicating the presence of ether linkage. Products were purified by column chromatography in 100-200 mesh silica. The product gave a single spot on TLC plate. All the synthesized compounds were characterized by ${ }^{1} \mathrm{H}-\mathrm{NMR},{ }^{13} \mathrm{C}-\mathrm{NMR}$ and FT-IR spectral data.

\section{General procedure for synthesis of $\beta$-amino alcohols derivatives:}

A mixture of phenols 1a-l (2 mmol), epichlorohydrin $(3 \mathrm{mmol})$, lipase $(10 \% \mathrm{w} / \mathrm{w}),[\mathrm{BMIM}] \mathrm{Cl}(0.2$ $\mathrm{mmol})$ and $\mathrm{K}_{2} \mathrm{CO}_{3}(2 \mathrm{mmol})$ was stirred in $25 \mathrm{ml}$ round bottom flask at $55{ }^{\circ} \mathrm{C}$ till the consumption of phenol (confirmed by TLC). Amine 2a-l $(2 \mathrm{mmol})$ was then added in one portion to same reaction mixture and stirred at $55^{\circ} \mathrm{C}$ to complete the reaction. The progress of the reaction was monitored by TLC. After completion of the reaction, it was then cooled to room temperature, then added ethyl acetate $(10 \mathrm{ml})$ and water $(10 \mathrm{ml})$.

Lipase was then filtered and then ethyl acetate layer was separated from water layer. It was dried by using anhydrous $\mathrm{Na}_{2} \mathrm{SO}_{4}$ and concentrated in high vacuum to give the final crude product. Products were purified by column chromatography on 100-200 mesh silica compound eluted in ethyl acetate:hexane (6:4) to afford the pure final product. The separated lipase was washed with water, dried at room temperature and reused for the same reaction.

\section{General procedure for synthesis of 1-butyl-3-methylimidazolium chloride ${ }^{43}$ ([BMIM]CI):}

A mixture of 1-methylimidazole $(1 \mathrm{mmol})$ and butyl chloride $(1.2 \mathrm{mmol})$ were stirred in round bottom flask fitted with a reflux condenser. The reaction mixture was refluxed for $12 \mathrm{~h}$ at $120{ }^{\circ} \mathrm{C}$ with constant stirring to complete the reaction by TLC. After completion, the reaction mass was cooled to room temperature, and the unreacted starting material was removed by distillation in high vacuum at $70{ }^{\circ} \mathrm{C}$ and $300 \mathrm{~atm}$ pressure to get final [BMIM]Cl. It was used as PTC in synthesis of $\beta$-amino alcohols derivatives.

\section{Spectra data}

1-Morpholino-3-phenoxypropan-2-ol, Table-3, Entry 3a:

A yellowish brown colored liquid.

FT-IR (neat, $\mathrm{cm}^{-1}$ ): 3402, 2923, 2858, 1593, 1492, 1456, 1240, 1110, 1039, 865, 802, 754, 688.

${ }^{1}$ H NMR (300 MHz, $\left.\mathrm{CDCl}_{3}\right): 2.35-2.75(\mathrm{~m}, 6 \mathrm{H}), 3.60-3.80(\mathrm{~m}, 5 \mathrm{H}), 3.98(\mathrm{~d}, 2 \mathrm{H}, J=5.1 \mathrm{~Hz}), 4.10(\mathrm{~m}$, $1 \mathrm{H}), 6.88-7.00$ (q, $J=7.2 \mathrm{~Hz}, 7.5 \mathrm{~Hz}, 2 \mathrm{H}), 7.22-7.34$ (q, $J=7.2 \mathrm{~Hz}, 7.8 \mathrm{~Hz}, 8.4 \mathrm{~Hz}, 3 \mathrm{H})$.

${ }^{13}$ C NMR (75 MHz, $\mathrm{CDCl}_{3}$ ): $\delta$ (ppm) 53.8, 61.1, 65.5, 66.9, 70.1, 114.5, 121.0, 129.5, 158.6.

\section{1-Morpholino-3-(naphthalen-1-yloxy)propan-2-ol, Table-3, Entry 3b:}

A yellow colored liquid.

FT-IR (neat, $\mathrm{cm}^{-1}$ ): 3413, 2925, 2858, 1583, 1452, 1396, 1269, 1105, 1008, 864, 779.

${ }^{1}$ H NMR (300 MHz, CDCl $)$ ) 2.40-3.05 (m, 7H), 3.65-3.85 (m, 4H), 4.05-4.35 (m, 3H), $6.82(\mathrm{~d}, J=$ $7.5 \mathrm{~Hz}, 1 \mathrm{H}), 7.32-7.54(\mathrm{~m}, J=3.9 \mathrm{~Hz}, 5.4 \mathrm{~Hz}, 7.2 \mathrm{~Hz}, 4 \mathrm{H}), 7.80$ (dd, $J=3.3 \mathrm{~Hz}, 7.2 \mathrm{~Hz}, 1 \mathrm{H}), 8.26$ (t, $J=5.4 \mathrm{~Hz}, 7.2 \mathrm{~Hz}, 9.6 \mathrm{~Hz}, 1 \mathrm{H})$.

${ }^{13}$ C NMR (75 MHz, $\left.\mathrm{CDCl}_{3}\right): \delta$ (ppm) 53.7, 61.3, 65.6, 66.9, 70.4, 104.8, 120.6, 121.8, 125.2, 125.5, $125.8,126.4,127.5,134.4,154.3$.

1-(Isopropylamino)-3-(naphthalen-1-yloxy)propan-2-ol, Table-3, Entry 3c:

A yellow colored liquid.

FT-IR (neat, $\mathrm{cm}^{-1}$ ): 3328, 3056, 2962, 1581, 1454, 1394, 1269, 1099, 761. 
${ }^{1}$ H NMR $\left(300 \mathrm{MHz}, \mathrm{CDCl}_{3}\right): 1.05(\mathrm{~d}, 6 \mathrm{H}), 2.65-2.95(\mathrm{~m}, 2 \mathrm{H}), 3.05(\mathrm{~m}, 1 \mathrm{H}), 3.85(\mathrm{bs}, 1 \mathrm{H}), 4.10(\mathrm{~d}$, $2 \mathrm{H}, J=4.8 \mathrm{~Hz}), 4.21(\mathrm{~m}, 1 \mathrm{H}), 6.74(\mathrm{q}, J=7.5 \mathrm{~Hz}, 7.8 \mathrm{~Hz}, 10.8 \mathrm{~Hz}, 1 \mathrm{H}), 7.26-7.50(\mathrm{~m}, J=1.5 \mathrm{~Hz}$, $6.6 \mathrm{~Hz}, 7.8 \mathrm{~Hz}, 9.3 \mathrm{~Hz}, 10.8 \mathrm{~Hz}, 4 \mathrm{H}), 7.76(\mathrm{t}, J=9.3 \mathrm{~Hz}, 1 \mathrm{H}), 8.24(\mathrm{t}, J=6.6 \mathrm{~Hz}, 9.3 \mathrm{~Hz}, 1 \mathrm{H})$.

${ }^{13}$ C NMR $\left(75 \mathrm{MHz}, \mathrm{CDCl}_{3}\right): \delta(\mathrm{ppm}) 18.2,19.9,52.7,53.6,68.6,70.3,104.9,120.6,121.8,125.3$, $125.5,125.9,126.4,127.5,134.5,154.3$.

1-(Piperidin-1-yl)-3-(m-tolyloxy)propan-2-ol, Table-3, Entry 3d:

A yellowish orange colored liquid.

FT-IR (neat, $\mathrm{cm}^{-1}$ ): 3396, 2931, 2796, 1595, 1483, 1448, 1259, 1161, 1043, 993, 935, 864, 775, 686.

${ }^{1}$ H NMR $\left(300 \mathrm{MHz}, \mathrm{CDCl}_{3}\right): 1.32-1.64(\mathrm{~m}, 6 \mathrm{H}), 2.26(\mathrm{~s}, 3 \mathrm{H}), 2.26-2.64(\mathrm{~m}, 6 \mathrm{H}), 3.66(\mathrm{bs}, 1 \mathrm{H}), 3.89$ $(\mathrm{d}, 2 \mathrm{H}, J=5.1 \mathrm{~Hz}), 4.02(\mathrm{~m}, 1 \mathrm{H}), 6.63-6.74(\mathrm{t}, J=1.2 \mathrm{~Hz}, 7.2 \mathrm{~Hz}, 9.3 \mathrm{~Hz}, 3 \mathrm{H}), 7.09(\mathrm{t}, J=1.2 \mathrm{~Hz}, 3$ $\mathrm{Hz}, 1 \mathrm{H})$.

${ }^{13}$ C NMR (75 MHz, $\mathrm{CDCl}_{3}$ ): $\delta$ (ppm) 21.4, 24.1, 25.9, 54.7, 61.3, 65.4, 70.3, 111.3, 115.3, 121.6, $129.1,139.3,158.7$.

1-(Methylamino)-3-(m-tolyloxy)propan-2-ol, Table-3, Entry 3e:

A yellowish brown colored liquid.

FT-IR (neat, $\mathrm{cm}^{-1}$ ): 3375, 2916, 2804, 1595, 1452, 1257, 1161, 1043, 931, 867, 773, 686.

${ }^{1}$ H NMR $\left(300 \mathrm{MHz}, \mathrm{CDCl}_{3}\right): 2.28(\mathrm{~s}, 3 \mathrm{H}), 2.36(\mathrm{~d}, 1 \mathrm{H}), 2.50-2.74(\mathrm{~m}, 4 \mathrm{H}), 3.50(\mathrm{bs}, 1 \mathrm{H}), 3.91(\mathrm{~d}$, $2 \mathrm{H}, J=5.7 \mathrm{~Hz}), 4.08(\mathrm{~m}, 1 \mathrm{H}), 6.64-6.80(\mathrm{q}, J=7.8 \mathrm{~Hz}, 8.1 \mathrm{~Hz}, 9.3 \mathrm{~Hz}, 3 \mathrm{H}), 7.08-7.17$ (t, $J=7.8 \mathrm{~Hz}$, $8.1 \mathrm{~Hz}, 1 \mathrm{H})$.

${ }^{13}$ C NMR (75 MHz, $\left.\mathrm{CDCl}_{3}\right): \delta$ (ppm) 21.5, 42.9, 60.5, 67.4, 70.1, 111.4, 115.4, 121.8, 129.2, 139.5, 158.6 .

1-(2-Chlorophenoxy)-3-morpholinopropan-2-ol, Table-3, Entry 3f:

A brown colored liquid.

FT-IR (neat, $\mathrm{cm}^{-1}$ ): 3407, 2950, 2815, 1585, 1481, 1450, 1284, 1244, 1112, 1062, 865, 750, 692.

${ }^{1}$ H NMR $\left(300 \mathrm{MHz}, \mathrm{CDCl}_{3}\right): 2.42-2.72(\mathrm{~m}, 6 \mathrm{H}), 3.38(\mathrm{~s}, 1 \mathrm{H}), 3.64-3.78(\mathrm{~m}, 4 \mathrm{H}), 4.04(\mathrm{~d}, 2 \mathrm{H}, J=4.8$ $\mathrm{Hz}), 4.14(\mathrm{~m}, 1 \mathrm{H}), 6.85-6.98(\mathrm{~m}, J=1.5 \mathrm{~Hz}, 6.9 \mathrm{~Hz}, 7.5 \mathrm{~Hz}, 7.8 \mathrm{~Hz}, 2 \mathrm{H}), 7.19(\mathrm{~m}, J=1.8 \mathrm{~Hz}, 6.6 \mathrm{~Hz}$, $7.5 \mathrm{~Hz}, 7.8 \mathrm{~Hz}, 1 \mathrm{H}), 7.34$ (dd, $J=1.5 \mathrm{~Hz}, 1.8 \mathrm{~Hz}, 6.0 \mathrm{~Hz}, 7.5 \mathrm{~Hz}, 7.8 \mathrm{~Hz}, 1 \mathrm{H})$.

${ }^{13}$ C NMR (75 MHz, $\left.\mathrm{CDCl}_{3}\right): \delta(\mathrm{ppm})$ 53.7, 60.9, 65.6, 66.8, 71.3, 113.7, 121.7, 122.9, 127.7, 130.2, 154.1 .

1-(2-Chlorophenoxy)-3-(isopropylamino)propan-2-ol, Table-3, Entry 3g:

A yellow colored liquid.

FT-IR (neat, $\mathrm{cm}^{-1}$ ): 3357, 2962, 2877, 1585, 1477, 1245, 1056, 1027, 935, 813, 746, 690

${ }^{1}$ H NMR $\left(300 \mathrm{MHz}, \mathrm{CDCl}_{3}\right): 1.05(\mathrm{~d}, 6 \mathrm{H}), 2.60-2.90(\mathrm{~m}, 2 \mathrm{H}), 3.05(\mathrm{~m}, 1 \mathrm{H}), 3.85-4.15(\mathrm{~m}, 4 \mathrm{H}), 6.83-$ $6.96(\mathrm{~m}, J=1.5 \mathrm{~Hz}, 7.5 \mathrm{~Hz}, 7.8 \mathrm{~Hz}, 9.6 \mathrm{~Hz}, 2 \mathrm{H}), 7.17$ (t, $J=1.8 \mathrm{~Hz}, 7.2 \mathrm{~Hz}, 7.5 \mathrm{~Hz}, 8.4 \mathrm{~Hz}, 9.3 \mathrm{~Hz}$, $1 \mathrm{H}), 7.33(\mathrm{dd}, J=1.5 \mathrm{~Hz}, 7.8 \mathrm{~Hz}, 9.3 \mathrm{~Hz}, 1 \mathrm{H})$.

${ }^{13}$ C NMR (75 MHz, $\left.\mathrm{CDCl}_{3}\right): \delta(\mathrm{ppm}) 17.9,19.5,52.7,53.3,68.2,70.9,113.7,121.7,122.8,127.7$, 130.1, 154.0.

1-(4-Bromophenoxy)-3-(methylamino)propan-2-ol, Table-3, Entry 3h:

A yellow colored liquid.

FT-IR (neat, $\mathrm{cm}^{-1}$ ): 3386, 2933, 2804, 1585, 1483, 1236, 1033, 881, 815, 690.

${ }^{1}$ H NMR $\left(300 \mathrm{MHz} \mathrm{CDCl}_{3}\right): 2.38(\mathrm{~d}, 1 \mathrm{H}), 2.52-2.74(\mathrm{~m}, 4 \mathrm{H}), 3.44(\mathrm{bs}, 1 \mathrm{H}), 3.90(\mathrm{~d}, 2 \mathrm{H}, J=4.8$ $\mathrm{Hz}), 4.10(\mathrm{~m}, 1 \mathrm{H}), 6.76$ (dd, $J=2.1 \mathrm{~Hz}, 6.9 \mathrm{~Hz}, 8.1 \mathrm{~Hz}, 2 \mathrm{H}), 7.35$ (dd, $J=1.8 \mathrm{~Hz}, 6.9 \mathrm{~Hz}, 8.7 \mathrm{~Hz}$, $2 \mathrm{H})$.

${ }^{13}$ C NMR (75 MHz, $\left.\mathrm{CDCl}_{3}\right): \delta(\mathrm{ppm}) 42.8,60.2,67.2,70.3,113.2,116.3,132.2,157.6$.

4-(2-Hydroxy-3-(isopropylamino)propoxy)benzonitrile, Table-3, Entry 3i:

A yellow colored liquid.

FT-IR (neat, $\mathrm{cm}^{-1}$ ): 3404, 2962, 2358, 2223, 1602, 1504, 1458, 1253, 1170, 1101, 1022, 831, 715. 
${ }^{1}$ H NMR (300 MHz, $\left.\mathrm{CDCl}_{3}\right)$ : $1.06(\mathrm{~d}, J=6.6 \mathrm{~Hz}, 6 \mathrm{H}), 2.56-2.84(\mathrm{~m}, 2 \mathrm{H}), 3.00(\mathrm{~m}, 1 \mathrm{H}), 3.46$ (bs, $1 \mathrm{H}), 3.96-4.14(\mathrm{~m}, 3 \mathrm{H}), 6.96(\mathrm{dd}, J=1.8 \mathrm{~Hz}, 2.1 \mathrm{~Hz}, 6.9 \mathrm{~Hz}, 9 \mathrm{~Hz}, 2 \mathrm{H}), 7.56(\mathrm{dd}, J=1.8 \mathrm{~Hz}, 2.4 \mathrm{~Hz}$, $6.9 \mathrm{~Hz}, 9.6 \mathrm{~Hz}, 2 \mathrm{H})$.

${ }^{13}$ C NMR (75 MHz, $\left.\mathrm{CDCl}_{3}\right): \delta(\mathrm{ppm}) 18.1,19.8,52.3,52.9,68.1,70.4,103.9,115.2,119.0,133.9$, 161.9 .

4-(3-(t-Butylamino)-2-hydroxypropoxy)benzonitrile, Table-3, Entry 3j:

A white solid.

MP: $99-101^{\circ} \mathrm{C}$

FT-IR (neat, $\mathrm{cm}^{-1}$ ): 3132, 2925, 2860, 2219, 1596, 1500, 1251, 1120, 1016, 916, 837, 707.

${ }^{1}$ H NMR $\left(300 \mathrm{MHz}, \mathrm{CDCl}_{3}\right): 1.14(\mathrm{~s}, 9 \mathrm{H}), 2.70(\mathrm{~m}, 1 \mathrm{H}), 2.86(\mathrm{dd}, 1 \mathrm{H}), 3.46(\mathrm{bs}, 1 \mathrm{H}), 4.05(\mathrm{~m}, 3 \mathrm{H})$, $6.98(\mathrm{dd}, J=2.1 \mathrm{~Hz}, 6.6 \mathrm{~Hz}, 8.7 \mathrm{~Hz}, 2 \mathrm{H}), 7.57(\mathrm{dd}, J=2.1 \mathrm{~Hz}, 6.9 \mathrm{~Hz}, 8.7 \mathrm{~Hz}, 2 \mathrm{H})$.

${ }^{13} \mathrm{C}$ NMR (75 MHz, $\left.\mathrm{CDCl}_{3}\right): \delta$ (ppm) 28.6, 28.8, 44.6, 50.9, 68.0, 70.7, 104.1, 115.3, 119.1, 133.9, 162.0 .

1-(t-Butylamino)-3-(4-nitrophenoxy)propan-2-ol, Table-3, Entry 3k:

A yellowish orange colored liquid.

FT-IR (neat, $\mathrm{cm}^{-1}$ ): 3299, 2964, 1593, 1506, 1334, 1259, 1107, 1020, 850, 750, 690.

${ }^{1}$ H NMR (300 MHz, CDCl $): 1.15$ (s, 9H), 2.75 (q, 1H), 2.90(dd, 1H), 4.00-4.15 (m, 3H), 4.25 (bs, $1 \mathrm{H}), 6.98(\mathrm{dd}, J=1.8 \mathrm{~Hz}, 6.9 \mathrm{~Hz}, 9 \mathrm{~Hz}, 2 \mathrm{H}), 8.18(\mathrm{dd}, J=1.8 \mathrm{~Hz}, 7.2 \mathrm{~Hz}, 9.3 \mathrm{~Hz}, 2 \mathrm{H})$.

${ }^{13}$ C NMR (75 MHz, $\left.\mathrm{CDCl}_{3}\right): \delta(\mathrm{ppm}) 28.5,44.6,51.3,67.9,71.2,114.5,125.8,141.5,163.7$.

1-Morpholino-3-(4-nitrophenoxy)propan-2-ol, Table-3, Entry 31:

A white solid.

MP: $78-80^{\circ} \mathrm{C}$

FT-IR (neat, $\mathrm{cm}^{-1}$ ): 3271, 2937, 2827, 1587, 1498, 1330, 1253, 1105, 989, 898, 850, 748.

${ }^{1}$ H NMR $\left(300 \mathrm{MHz}, \mathrm{CDCl}_{3}\right):$ 2.44-2.76 (m, 6H), $3.32(\mathrm{~s}, 1 \mathrm{H}), 3.68-3.82(\mathrm{~m}, 4 \mathrm{H}), 4.02-4.20(\mathrm{~m}, 3 \mathrm{H})$, $7.00(\mathrm{dd}, J=2.1 \mathrm{~Hz}, 3.3 \mathrm{~Hz}, 6.9 \mathrm{~Hz}, 7.2 \mathrm{~Hz}, 9.3 \mathrm{~Hz}, 2 \mathrm{H}), 8.19(\mathrm{dd}, J=2.1 \mathrm{~Hz}, 3.3 \mathrm{~Hz}, 7.2 \mathrm{~Hz}, 9.3$ $\mathrm{Hz}, 2 \mathrm{H})$.

${ }^{13}$ C NMR (75 MHz, $\left.\mathrm{CDCl}_{3}\right): \delta(\mathrm{ppm})$ 53.7, 60.7, 65.1, 66.8, 70.9, 114.5, 125.8, 141.6, 163.7.

\section{Acknowledgments}

The Authors are thankful to University Grant Commission (UGC-SAP programme) INDIA for financial assistance to Mr. Vasant S. Borude and Mr. Rikhil V. Shah.

\section{References}

1. Erhardt, P. W., WOO, C. M., Gorczynski, R. J., and Anderson, W. G. (1982) Ultra-shortacting $\beta$-adrenergic receptor blocking agents. 1. (Aryloxy) propanolamines containing esters in the nitrogen substituent. J. Med. Chem., 25, 1402-1407.

2. Kopka, K., Wagner, S., Riemann, B., Law, M. P., Puke, C., Luthra, S. K., Pike, V. W., Wichter, T., Schmitz, W., Schober, O., and Schafers, M. (2003) Design of new $\beta_{1}$-selective adrenoceptor ligands as potential radioligands for in vivo imaging. Bioorg. Med. Chem., 11, 3513-3527.

3. Slater, R. A., Howson, W., Swayne, G. T. G., Taylor, E. M., and Reavill, D. R. (1988) Design and synthesis of a series of combined vasodilator/ $\beta$-adrenoceptor antagonists based on 6-arylpyridazinones. J. Med. Chem., 31, 345-351.

4. Gallego, L., and Arroyo, P. (2003) Determination of prednisolone, naphazoline, and phenylephrine in local pharmaceutical preparations by micellar electrokinetic chromatography. J. Sep. Sci., 26, 947-952. 
5. Klingler, F. D. (2007) Asymmetric hydrogenation of prochiral amino ketones to amino alcohols for pharmaceutical use. Acc. Chem. Res., 40, 1367-1376.

6. Castellano, M., and Bohm, M. (1997) The cardiac $\beta$-adrenoceptor-mediated signaling pathway and its alterations in hypertensive heart disease. Hypertension, 29, 715-722.

7. Yamada, S., Ohkura, T., Uchida, S., Inabe, K., Iwatani, Y., Kimura, R., Hoshino, T., and Kaburagi, T. (1996) A sustained increase in beta-adrenoceptors during long-term therapy with metoprolol and bisoprolol in patients with heart failure from idiopathic dilated cardiomyopathy. Life Sci., 58, 1737-1744.

8. Auvin-Guette, C., Rebuffat, S., Prigent, Y., and Bodo, B. (1992) Trichogin A IV, an 11Residue lipopeptaibol from trichoderma longibrachiatum. J. Am. Chem. Soc., 114, 2170-2174.

9. Wright, J. L., Gregory, T. F., Heffner, T. G., Mackenzie, R. G., Pugsley, T. A., Meulen, S. V., and Wise, L. D. (1997) Discovery of selective dopamine $\mathrm{d}_{4}$ receptor antagonists: 1-aryloxy-3(4-aryloxypiperidinyl)-2-propanols. Bioorg. Med. Chem., 7, 1377-1380.

10. Marquis, R. W., Lago, A. M., Callahan, J. F., Lee-Trout, R. E., Gowen, M., DelMar, E. G., Van-Wagenen, B. C., Logan, S., Shimizu, S., Fox, J., Nemeth, E. F., Yang, Z., Roethke, T., Smith, B. R., Ward, K. W., Lee, J., Keenan, R. M., and Bhatnagar, P. (2009) Antagonists of the calcium receptor I. Amino alcohol-based parathyroid hormone secretagogues. J. Med. Chem., 52, 3982-3993.

11. Apparu, M., Tiba, Y. B., Leo, P., Hamman, S., and Coulombeau, C. (2000) Determination of the enantiomeric purity and the configuration of $\beta$-amino alcohols using (R)-2fluorophenylacetic acid (AFPA) and fluorine-19 NMR: application to $\beta$-blockers. Tetrahedron Asymmetry, 11, 2885-2898.

12. Joshi, R. A., Gurjar, M. K., and Tripathy, N. K. (2001) A new and improved process for celiprolol hydrochloride. Org. Process Res. Dev., 5, 176-178.

13. Cepanec, I., Litvic, M., Mikuldas, H., Bartolincic, A., and Vinkovic, V. (2003) Calcium trifluoromethanesulfonate-catalysed aminolysis of epoxides. Tetrahedron, 59, 2435-2439.

14. Yadav, J. S., Reddy, B. V. S., Basak, A. K., and Narsaiah, A. V. (2003) $\left[\mathrm{Bmim}^{\mathrm{B}} \mathrm{BF}_{4}\right.$ ionic liquid: a novel reaction medium for the synthesis of $\beta$-amino alcohols. Tetrahedron Lett., 44, 1047-1050.

15. Ollevier, T., and Lavie-Compin, G. (2004) Bismuth triflate-catalyzed mild and efficient epoxide opening by aromatic amines under aqueous conditions. Tetrahedron Lett., 45, 49-52.

16. Peukert, S., and Jacobsen, E. N. (1999) Enantioselective parallel synthesis using polymersupported chiral Co(salen) complexes. Org. Lett., 1, 1245-1248.

17. Kantam, M. L., Neelima, B., Reddy, V., and Chakravarti, R. (2007) Aza-michael addition of imidazoles to $\alpha, \beta$-unsaturated compounds and synthesis of $\beta$-amino alcohols via nucleophilic ring opening of epoxides using copper(II)acetylacetonate $\left(\mathrm{Cu}(\mathrm{acac})_{2}\right)$ immobilized in ionic liquids. Ind. Eng. Chem. Res., 46, 8614-8619.

18. Mojtahedi, M. M., Saidi, M. R., and Bolourtchian, M. (1999) Microwave-assisted aminolysis of epoxides under solvent-free conditions catalyzed by montmorillonite clay. J. Chem. Res. (S)., 128-129.

19. Pchelka, B. K., Loupy, A., and Petit, A. (2006) Improvement and simplification of synthesis of 3-aryloxy-1,2-epoxypropanes using solvent-free conditions and microwave irradiations. Relation with medium effects and reaction mechanism. Tetrahedron, 62, 10968-10979.

20. Kazuhiro, K., Yoshiro, F., Hiroshi, Y., and Junzo, O. (1999) CsF in organic synthesis. Regioselective nucleophilic reactions of phenols with oxiranes leading to enantiopure $\beta$ blockers. Tetrahedron, 55, 14381-14390.

21. Fisher, L. G., Sher, P. M., Skwish, S., Michel, I. M., Seiler, S. M., and Dickinson, K. E. (1996) BMS-187257, a potent, selective, and novel heterocyclic $\beta_{3}$-adrenergic receptor agonist. J. Bioorg. Med. Chem. Lett., 6, 2253-2258.

22. Sun, D., Scherman, M. S., Jones, V., Hurdle, J. G., Woolhiser, L. K., Knudson, S. E., Lenaerts, A. J., Slayden, R. A., McNeil, M. R., and Lee, R. E. (2009) Discovery, synthesis, 
and biological evaluation of piperidinol analogs with anti-tuberculosis activity. Bioorg. Med. Chem., 17, 3588-3594.

23. Surendra, K., Srilakshmi-Krishnaveni, N., and Rama-Rao, K. (2005) The selective C-3 opening of aromatic 2,3-epoxy alcohols/epoxides with aromatic amines catalysed by $\beta$ cyclodextrin in water. Synlett, 3, 506-510.

24. Klibanov, A. M. (1986) Enzymes that work in organic solvents. Chemtech, 16, 354-359.

25. Pyo, S. H., Persson, P., Lundmark, S., and Hatti-Kaul, R. (2011) Solvent-free lipase-mediated synthesis of six-membered cyclic carbonates from trimethylolpropane and dialkyl carbonates. Green chem., 13, 976-982.

26. Sonawane, Y. A., Phadtare, S. B., Borse, B. N., Jagtap, A. R., and Shankarling, G. S. (2010) Synthesis of diphenylamine-based novel fluorescent styryl colorants by knoevenagel condensation using a conventional method, biocatalyst, and deep eutectic solvent. Org. Lett., $12,1456-1459$.

27. Li, K., He, T., Li, C., Feng, X. W., Wang, N., and Yu, X. Q. (2009) Lipase-catalysed direct mannich reaction in water: utilization of biocatalytic promiscuity for $\mathrm{C}-\mathrm{C}$ bond formation in a one-pot synthesis. Green Chem., 11, 777-779.

28. Fang, J. M., and Wong, C. H. (1994) Enzymes in organic synthesis: alteration of reversible reactions to irreversible processes. Synlett, 6, 393-402.

29. Starks, C. M. (1971) Phase-transfer catalysis. I. Heterogeneous reactions involving anion transfer by quaternary ammonium and phosphonium salts. J. Am. Chem. Soc., 93, 195-199.

30. Hashimoto, T., and Maruoka, K. (2007) Recent development and application of chiral phasetransfer catalysts. Chem. Rev., 107, 5656-5682.

31. Hajipour, A. R., and Rafiee, F. (2009) Basic ionic liquids. A short review. J. Iran. Chem. Soc., 6, 647-678.

32. Ranu, B. C., and Banerjee, S. (2005) Ionic liquid as catalyst and reaction medium. The dramatic influence of a task-specific ionic liquid, [bmIm] $\mathrm{OH}$, in michael addition of active methylene compounds to conjugated ketones, carboxylic esters, and nitriles. Org. Lett., 7, 3049-3052.

33. Jiang, D., Wang, Y., Sun, H., and Dai, L. Y., (2007) Phase transfer reaction catalyzed by ionic liquids. J. Chil. Chem. Soc., 52, 1302-1304.

34. Yadav, G. D., and Motirale, B. G. (2008) Ionic liquid as catalyst for solid-liquid phase transfer catalyzed synthesis of p-nitrodiphenyl ether. Ind. Eng. Chem. Res., 47, 9081-9089.

35. Kim, D. W., and Chi, D. Y. (2004) Polymer-supported ionic liquids: imidazolium salts as catalysts for nucleophilic substitution reactions including fluorinations. Angew. Chem. Int. Ed., 43, 483-485;

36. Kim, D. W., Song, C. E., and Chi, D. Y. (2003) Significantly enhanced reactivities of the nucleophilic substitution reactions in ionic liquid. J. Org. Chem., 68, 4281-4285.

37. Xie, H., Zhang, S., and Duan, H. (2004) An ionic liquid based on a cyclic guanidinium cation is an efficient medium for the selective oxidation of benzyl alcohols. Tetrahedron Lett., 45, 2013-2015.

38. Kryshtal, G. V., Zhdankina, G. M., and Zlotin S. G. (2005) Tetraalkylammonium and 1,3dialkylimidazolium salts with fluorinated anions as recoverable phase-transfer catalysts in solid base-promoted cross-aldol condensations. Eur. J. Org. Chem., 2822-2827.

39. Azizi, N., and Saidi, M. R. (2005) Highly chemoselective addition of amines to epoxides in water. Org. Lett., 7, 3649-3651.

40. Khadilkar, B. M., and Bendale, P. M. (1997) Microwave enhanced synthesis of epoxypropoxyphenols. Synth. Commun., 27, 2051-2056.

41. Borse, B. N., Borude, V. S., and Shukla, S. R. (2012) Synthesis of novel dihydropyrimidin$2(1 \mathrm{H})$-ones derivatives using lipase and their antimicrobial activity. Curr. Chem. Lett. 1, 4758. 
42. Harris, T. K., and Turner, G. J. (2002) Structural Basis of Perturbed $\mathrm{pK}_{\mathrm{a}}$ Values of Catalytic Groups in Enzyme Active Sites. IUBMB Life, 53, 85-98.

43. Palimkar, S. S., Siddiqui, S. A., Daniel, T., Lahoti R. J. and Srinivasan, K. V. (2003) Ionic liquid-promoted regiospecific friedlander annulation: novel synthesis of quinolines and fused polycyclic quinolines. J. Org. Chem., 68, 9371-9378. 